\title{
Indexing of Ears using Radial basis Function Neural Network for Personal Identification
}

\author{
M.A. Jayaram ${ }^{1}$ \\ ${ }^{1}$ Director, \\ Department of Master of Computer \\ Applications \\ Siddaganga Institute of Technology, \\ Tumkur \\ karnataka, India
}

\author{
Prashanth G.K ${ }^{2}$. \\ ${ }^{2}$ Assistant Professor, \\ Department of Master of Computer \\ Applications \\ Siddaganga Institute of Technology, \\ Tumkur \\ karnataka, India
}

\author{
M.Anusha ${ }^{3}$, \\ ${ }^{3}$ Project Student \\ Department of Master of Computer \\ Applications \\ Siddaganga Institute of Technology, \\ Tumkur \\ karnataka, India
}

\begin{abstract}
This paper elaborates a novel method to recognize persons using ear biometrics. We propose a method to index the ears using Radial Basis Function Neural Networks (RBFNN). In order to obtain the invariant features, an ear has been considered as a planar surface of irregular shape. The shape based features like planar area, moment of inertia with respect to minor and major axes, and radii of gyration with respect to minor and major axes are considered. The indexing equation is generated using the weights, centroids and kernel function of the stabilized RBFN network. The so developed indexing equation was tested and validated. The analysis of the equation revealed $95.4 \%$ recognition accuracy. The retrieval rate of personal details became faster by an average of $13.8 \%$ when the database was organized as per the indices. Further, the three groups elicited by RBFNN were evaluated for parameters like entropy, precision, recall, specificity and F-measure. And all the parameters are found to be excellent in terms of their values and thus showcase the adequacy of the indexing model.
\end{abstract}

Keywords-RBFNN; kernel function; Indexing equation; Moment of inertia; radii of gyration

\section{INTRODUCTION}

Alfred Iannarelli developed a new class of biometrics, based upon ear features and introduced it for use in the development of passive identification systems [1]. Identification by ear biometrics is promising because it is passive like face recognition. The ear is considered to be a unique feature for human beings. Even the ears of "identical twins" differ in some respects [2]. Unlike face, the configuration of ear will never be subjected to changes associated with changes in the facial expression, and the make-up effects. The configuration and the complexion of the ear do not vary with age. It has the biometric traits like uniqueness, universality, permanence and collectability.

A profound work of ear identification involving over 10000 ears has been documented [1]. In an experiment involving larger datasets more rigorously controlled for relative quality of face and ear, the recognition performance was almost same when ear and face were individually considered. However, the performance shot up to $90.9 \%$ when both ear and face were considered [3].

Ear biometrics is an unexplored biometric field, but has received a growing amount of attention over the past few years. There are three modes of ear biometrics: ear photographs, ear prints obtained by pressing the ear against a flat plane, and thermograph pictures of the ear. The most common implementation of ear biometrics is via photographs for identification systems [4].

This paper presents a novel method to index years by using radial basis function neural networks. By indexing, we mean provide an integer number to an Ear indicating to what group it belongs. This integer number will have evolved through RBFNN centroids, the kernel function and the weights of the stabilized network. The rest of the paper is organized as follows. Use of RBFNN in allied areas of biometrics is discussed in section II. The shape based biometrics developed by authors is presented in section III. Section IV presents about the data used in the model. A brief presentation of RBFNN is done in section V. The indexing equation is illustrated in section VI. Analysis of the results is elaborated in section VII. Section VIII concludes the paper.

\section{RELATED WORKS}

Considerable amount of research has gone into identifying methods suitable for indexing and classification of entities. The application of RBFNN in the domain of biometrics has been scarce. Mai $\mathrm{V}$ et al [5] proposed a new method to identify people using Electrocardiogram (ECG). QRS complex ( $\mathrm{Q}$ waves, $\mathrm{R}$ waves, $\mathrm{S}$ waves) which is a stable parameter against heart rate variability is used as a biometric feature. This work has reported for having achieved a classification accuracy of $97 \%$ using RBF.

Sulong et al [6] have used a combination of maximum pressure exerted on the keyboard and the time latency between the keystrokes to recognize the authenticate users and to reject imposters. In this work, RBFNN is used as a pattern matching method. The system so developed has been evaluated using False Reject Rate (FRR) and False Accept Rate (FAR). The researchers have affirmed the effectiveness of the security system designed by them.

Chatterjee et al [7] have proposed a new biometric system which is based on four types of temporal postural signals. The system employs S-transform to determine the characteristic features for each human posture. An RBFNN with these characteristic features as input is developed for specific 
authentication. The training of the network has augmented extended Kalman filtering (EKF). The overall authentication accuracy of the system is reported to be of the order of $95 \%$.

In a study, multi-modal biometric consisting of fingerprint images and finger vein patterns were used to identify the authorized users after determining the class of users by RBFNN as a classifier. The parameters of the RBFNN were optimized using BAT algorithm. The performance of RBFNN was found to be superior when compared with KNN, Naïve Bayesian and non-optimized RBFNN classifier [8].

Ankit Chadha et al have used signature of persons for verification and authentication purpose. RBFNN was trained with sample images in the database. The network successfully identified the original images with the recognition accuracy of $80 \%$ for image sample size of 200 [9].

Handwriting recognition with features such as aspect ratio, end points, junction, loop, and stroke direction were used for recognition of writers [10]. The system used over 500 text lines from 20 writers. RBFNN showed a recognition accuracy of $95.5 \%$ when compared to backpropagation network.

An Optical Character Recognition (OCR) is developed for the recognition of the basic characters such as vowels and consonants for Kannada text. The system can handle different font size and font types. Features such as Hu's invariant moments and Zernike moments are extracted and RBFNN is used as a classifier to identify and classify the characters [11].

\section{SHAPE BASED BIOMETRICS}

In this work, the five shape based features of ears that were considered for classification are listed in the Table I. The details of feature extraction, authentication, their evaluation and the elaboration of a personal identification system developed are available in seminal work of authors [12]. However, for the sake of completeness, the features are explained in the following paragraphs.

The surface area of the ear is the projected area of the curved surface on a vertical plane. Moment of Inertia (MI) is the property of a planar surface which originates whenever one has to compute the moment of distributed load that varies linearly from the moment axis. Moment of Inertia is also viewed as a physical measure that signifies the shape of a planar surface and it is proved that by configuring the shape of planar surface and hence by altering the moment of inertia, the resistance of the planar surface against rotation with respect to a particular axis could be modulated or altered [13]. Therefore in this work, moment of inertia of ear surface with respect to two axes i.e. the major axis and the minor axis are considered to be the best biometric attributes that could capture the shape of irregular surface of the ear in a scientific way.

As far as features are concerned, major axis is the one which has the longest distance between the two points on the edge of the ear, the distance here is the maximum among point to point Euclidean distance. The minor axis is drawn in such way that it passes through tragus and is orthogonal to the major axis. Therefore, with different orientation of ears the orientation of major axis also changes. Being perpendicular to major axis, the orientation of minor axis is fixed.
The projected area is assumed to be formed out of segments. The area of an ear to the right side of the major axis is considered to be made out of six segments. Each of the segments thus subtends $30^{\circ}$ with respect to the point of the intersection of the major axis and minor axis. The extreme edge of a sector is assumed to be a circular arc. Thus converting each segment into a sector of circle of varying area. Typical ear edge with measurements is shown in Figure 1 .

The measurements are

- $\theta \rightarrow$ Inclination of the central radial axis of the segment with respect to minor axis (in degrees).

- $\mathrm{r} \rightarrow$ The length of the radial axis (in $\mathrm{mm}$ ).

The conversion of number of pixel into linear dimension (in $\mathrm{mm}$ ) was based on the resolution of the camera expressed in PPI (Pixel Per Inch). In this work 16Mega pixel camera, at 300 PPI was used. The computation of linear distance is straight farward $\mathrm{mm}=($ number of pixel $* 25.4) / \mathrm{PPI}[1$ inch $=25.4$ $\mathrm{mm}]$. With these measurements, the following parameters are computed.

Moment of inertia with respect to minor axis $\mathrm{I}_{\min }$

$$
I_{\min }=\sum_{i=1}^{6} a_{i} y_{i}^{2}
$$

Where $a_{i}$ is the area of a the $i^{\text {th }}$ segment and $y_{i}$ is the perpendicular distance of the centroid of the $i^{\text {th }}$ segment with respect to minor axis.

$$
\begin{gathered}
a_{i}=\Theta r^{2} \\
\mathrm{y}_{\mathrm{i}}=\mathrm{C} \sin \theta
\end{gathered}
$$

Here, $\mathrm{C}$ is the centroidal distance of the segment with respect to the intersection point of the axes, which is given by[14];

$$
C=\frac{2}{3} \frac{r \sin \theta}{\theta}
$$

Simlarly, moment of inertia with respect to major axis $I_{\max }$ , $\mathrm{x}_{\mathrm{i}}$ is the perpendicular distance of the centroid of the ith segment with respect to major axis.

$$
\begin{gathered}
\mathrm{I}_{\max }=\sum_{\mathrm{i}=1}^{6} \mathrm{a}_{\mathrm{i}} \mathrm{x}_{\mathrm{i}}^{2} \\
\text { Where } x_{i}=C \cos \theta
\end{gathered}
$$

From the computed values of moment of inertia and area of the ear surface, the radii of gyration with respect to minor axis(RGx)and major axis(RGy) were computed. The formulae for radii of gyration are given by[15].

$$
\begin{aligned}
& R G x=\sqrt{\frac{I_{\min }}{A}} \\
& R G y=\sqrt{\frac{I_{\max }}{A}}
\end{aligned}
$$

Where, $\mathrm{A}$ is the sum of areas of six segments.

$$
A=\sum_{i=1}^{6} a_{i}
$$

Radius of gyration is the distance from an axis at which the mass of a body may be assumed to be concentrated and at 
which the moment of inertia will be equal to the moment of inertia of the actual mass about the axis. Mathematically it is equal to the square root of the quotient of the moment of inertia and the area. The axis of inertia is unique to the shape. It serves as a unique reference line to preserve the orientation of the shape. The axis of least inertia (ALI) of a shape is defined as the line for which the integral of the square of the distances to points on the shape boundary is a minimum [16].

TABLE I. SHAPE BASED FEATURES FOR ClASSIFICATION

\begin{tabular}{ll}
\hline \hline Sl. No & Attributes \\
\hline 1 & Area $\left(\mathrm{mm}^{2}\right)$ \\
2 & Moment of Inertia Y $(\operatorname{Imax})\left(\mathrm{mm}^{4}\right)$ \\
3 & Radius of gyration Y $(\mathrm{RGy})(\mathrm{mm})$ \\
4 & Moment of Inertia X (Imin) $\left(\mathrm{mm}^{4}\right)$ \\
5 & Radius of gyration X (RGx) $(\mathrm{mm})$ \\
\hline \hline
\end{tabular}

\section{DATA FOR THE MODEL}

Ear images for this indexing work were acquired from the pupils of Siddaganga group of institutes. The subjects involved were mostly students and faculty numbering 605 . In each acquisition session, the subject sat approximately one meter away with the side of the face in front of the camera in outside environment without flash.

The images so obtained were resized in such a way that only ear portion covers the entire frame having pixel matrix.

The color images were converted into gray scale images followed by uniform distribution of brightness through histogram equalization technique. The delineation of outer edge of each ear was obtained using canny edge detection algorithm. The resulting edge was inverted to get a clear boundary shape of the ear. The conceptual presentation of the process involved is shown in Figure 2.

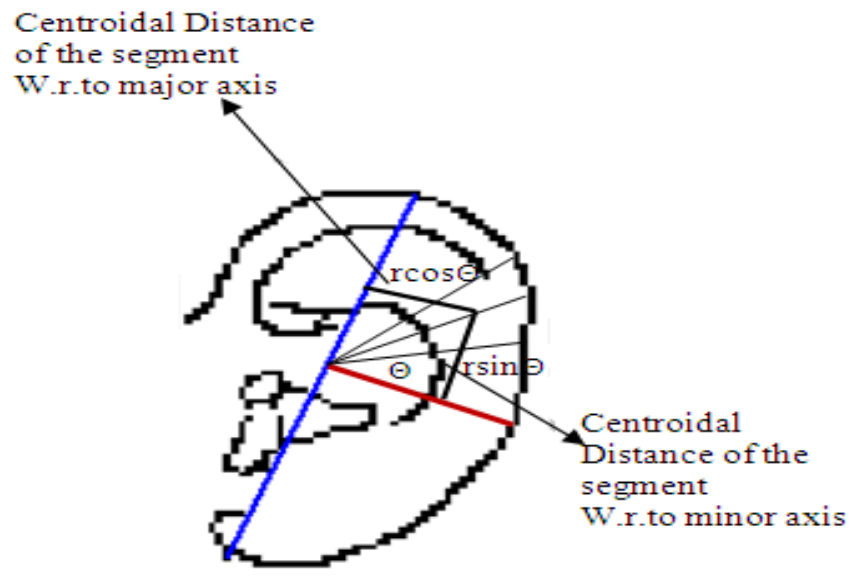

Fig. 1. Typical ear edge with M.I. parameters

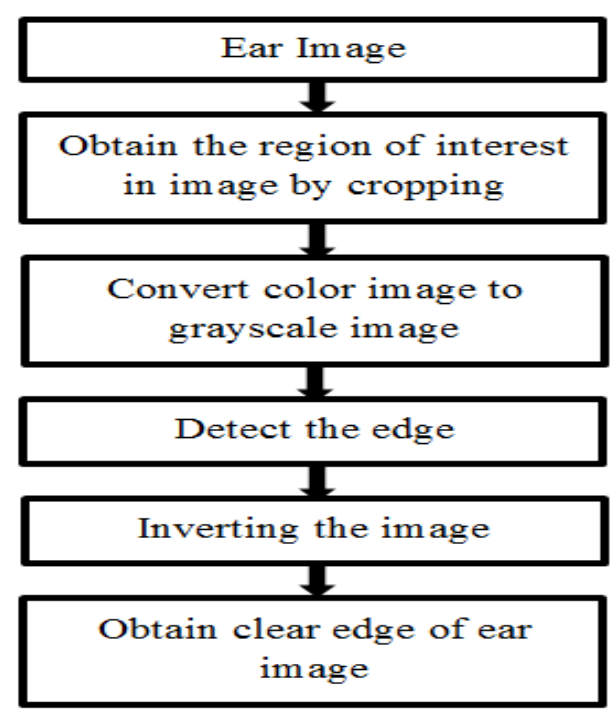

Fig. 2. The Steps involved in ear edge extraction

\section{RADIAL BASIS FUNCTION NEURAL NETWORKS}

A Radial Basis Function (RBF) network is a special type of neural network that uses a radial basis function as its activation function. RBF networks are very popular for function approximation, curve fitting, time series prediction, and classification problems. The radial basis function network is different from other neural networks, possessing several distinctive features. Because of their universal approximation, more compact topology and faster learning speed, RBF networks have been widely applied in many fields, in science and engineering.

The learning of RBFNN happens in three steps

- Finding the cluster centers of the radial basis function using the K-means clustering algorithm.

- Determining the width of the radial basis function.

- Computing the weights.

A block diagram of an RBF network used in this work is presented in Figure 3. The input layer is the 5-dimensional vector which has to be classified. The entire input vector is passed to each of the RBF neurons.

A prototype vector is stored by each RBF neuron which is just one of the vectors from the training set. Each RBF neuron compares the input vector to its prototype, and outputs a value between 0 and 1 which is a measure of similarity [17].

The output layer of the network consists of three nodes; one denoting each index. Each output node computes a score, upon which a classification decision is made by assigning the input to the highest output neuron score and affixing positional value of neuron as the index. The block diagram of the process of indexing is shown in Figure 4. 


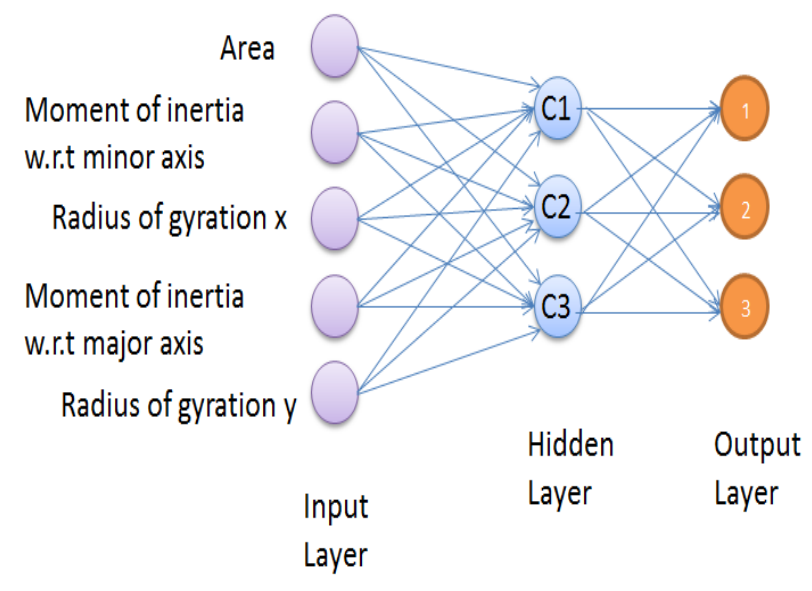

Fig. 3. Architecture of RBFNN

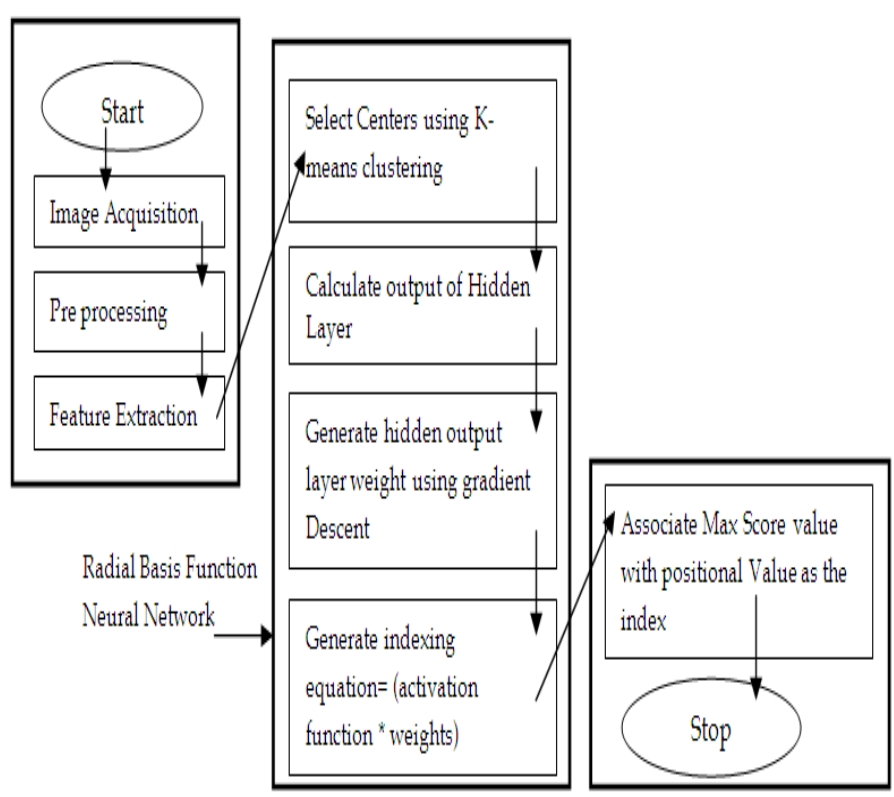

Fig. 4. Flowchart of the work

\section{A. K-means algorithm}

Initially, computational efforts were conducted in order to find appropriate number of clusters with minimum overlapping. It was found that three clusters were ideal as the overlapping were minimal. The corresponding centroid values for three classes as elicited by k-means algorithm are presented in Table II.

TABLE II. CENTROIDS OF Clusters as Determined By K-Means ALGORITHM

\begin{tabular}{llllll}
\hline \hline & Area & $\begin{array}{l}\text { Moment } \\
\text { of } \\
\text { Inertia X }\end{array}$ & $\begin{array}{l}\text { Radius of } \\
\text { gyration } \\
\text { X }\end{array}$ & $\begin{array}{l}\text { Moment } \\
\text { of Inertia } \\
\text { Y }\end{array}$ & $\begin{array}{l}\text { Radius of } \\
\text { gyration } \\
\text { Y }\end{array}$ \\
\hline $\begin{array}{l}\text { Group } \\
\text { I }\end{array}$ & 501.6093 & 6866031 & 118.308 & 1605.915 & 1.585156 \\
$\begin{array}{l}\text { Group } \\
\text { II }\end{array}$ & 396.3764 & 3798362 & 98.40404 & 684.1802 & 1.156876 \\
$\begin{array}{l}\text { Group } \\
\text { III }\end{array}$ & 270.4792 & 1666994 & 74.85014 & 292.8828 & 0.876999 \\
\hline \hline
\end{tabular}

\section{B. Gaussian activation function}

Each RBF neuron computes a measure of the similarity between the input and its prototype vector taken from the training set. Input vectors which are more similar to the prototype return a result closer to 1 . There are different possible kernel functions, but the most popular is based on the Gaussian which is given by

$$
\Phi=\exp \left[-\frac{\sum_{i=1}^{3}|| x-\left.\mu_{i}\right|^{2}}{2 \sigma^{2}}\right]
$$

Where $\mathrm{x}$ is the input pattern, $\mu$ is the centroid of RBF unit for input variables and $\sigma$ is the width of the RBF unit. Each RBF neuron will produce its largest response when the input is equal to the prototype vector. The linear sum of multiples of output of the central layer neurons and weights of connections will lead to outputs of the three neurons present in output layer.

\section{Gradient descent method}

The gradient descent method is applied for finding centers, spread and weights by minimizing the (instantaneous) squared error. Activation functions become the input to this method. The RBFNNs trained by the gradient-descent method is capable of providing the equivalent or better performance compared to that of the multi-layer feed forward neural network trained with the back propagation [18].

\section{THE INDEXING EQUATION}

Group Index (GI) formula is generated by using the centroids, the beta term and the output weights. Thus, the indexing equation is given by

$$
G I=\sum_{j=1}^{3} W_{j m} * \exp \left[-\frac{\sum_{i=1}^{3}|| x-\mu_{i} \|^{2}}{2 \sigma^{2}}\right]
$$

Where $\mathrm{x}$ is the input pattern, $\mu$ is the centroid of RBF unit for input variables and $\sigma$ is the width of the RBF unit, $\mathrm{W}$ is the weight between $j$ th RBF unit and $m$ th output node. 2-norm of a vector is used to normalize the entire dataset. The result of the successful run of the RBFNN is presented in Table VI for sample test set.

\section{RESULTS AND ANALYSIS}

Table III gives salient details of the network. The clusters using k-means and the result of RBFNN are presented in Table IV. This table indicates that KNN and RBFNN have emerged almost similar as far as the number of Ears that fall in to each category. In specific, group 1 has $2.5 \%$ more, group 2 has $7.5 \%$ less, and group 3 has $7.5 \%$ more number of ears. Thus RBFNN is instrumental in fine tuning the classification task rendered by KNN. The proposed indexing eqn. no (11) can be used to find the respective index value of the ear. To obtain this one has to substitute the feature values, centroid values, the weights and the kernel radius value indicated by $\sigma$ provided in Table III. Testing of the equation for around 200 ear images in the same database yielded almost $95.4 \%$ accuracy. The test image was searched in both unorganized database and the database organized in three consecutive blocks as per the indexes. The CPU time was measured for both the cases. It is found that, the average decrease in the 
CPU time was around $13.77 \%$ with organized database. This aspect of the model is shown in Table V, here again, 200 ear images were selected at random $\left(1 / 3^{\text {rd }}\right.$ of the database) and CPU time was noted. Justifiably, maximum time was consumed while matching and retrieving for the ears that fell in third category. Group characteristics are presented in Table VII A This table illustrates that though there are clear distinguishing features between groups as far are the appearance of the ear the attribute values overlap.

The contribution of this work lies in according an integer index to an ear based on certain moment of inertia related properties. By just looking at the index value of the ear, it is possible to get the notion of the articulation of the ear. To unearth this capability of indexing, the entire data base was studied with the ear image along with the attribute values and the corresponding index. Based on this empirical study it is possible to judge the group characteristic as shown in Table VII B. Classification performance evaluation measures are given in Table VIII and Table IX. An entropy value of 0.99395 indicates that the classes are more dispersed. An accuracy rate as high as $96 \%$ showcases an excellent classification. Precision values in the range $0.8-1$ of clusters determine high positive predictive value. Recall value in the range $0.9-1$ indicates that the clusters have high true positive rate. The value of specificity shows the high true-negative rate which is in the range $0.94-1$. F-measure concludes that there is a good balance between precision and recall in the second and third cluster.

TABLE III. SALIENT DETAILS OF RBFNN IMPLEMENTED

No of input layer neurons
No of neurons in hidden layer
The basis function
No of output neurons
Width of cluster as given by $\sigma$

\begin{tabular}{l|lll} 
C1 & C1 & C 2 & C 3 \\
\hline C2 & 0 & 8 & 0 \\
C3 & 9 & 11 & 247 \\
9
\end{tabular}

Fig. 5. Confusion matrix
TABLE IV. TEST SET AND GROUP INDEXING BY RBFNN

\begin{tabular}{|c|c|c|c|c|c|c|}
\hline $\begin{array}{l}\text { Sl. } \\
\text { No }\end{array}$ & Area & $\operatorname{Imax}$ & RGy & Imin & RGx & GI \\
\hline 1 & 131.3487 & 195355.9 & 38.56563 & 50.89822 & 0.622499 & 2 \\
\hline 2 & 135.0409 & 371368.5 & 52.44088 & 140.7316 & 1.020853 & 2 \\
\hline 3 & 404.5635 & 3158077 & 88.35233 & 766.4084 & 1.376375 & 3 \\
\hline 4 & 241.7773 & 744226.9 & 55.48108 & 116.0455 & 0.692798 & 2 \\
\hline 5 & 370.4741 & 2691695 & 85.23815 & 310.3129 & 0.91521 & 2 \\
\hline 6 & 272.2138 & 3054815 & 105.9345 & 0.344449 & 0.035572 & 3 \\
\hline 7 & 358.0337 & 3395618 & 97.38621 & 254.3965 & 0.842934 & 3 \\
\hline 8 & 369.2937 & 2464924 & 81.69882 & 264.376 & 0.846107 & 2 \\
\hline 9 & 217.2377 & 2858884 & 114.7178 & 11.01563 & 0.225184 & 3 \\
\hline 10 & 360.2648 & 2844322 & 88.8543 & 641.5627 & 1.334469 & 3 \\
\hline 11 & 338.5039 & 1991710 & 76.70634 & 368.703 & 1.043654 & 2 \\
\hline 12 & 379.9424 & 5368573 & 118.8695 & 240.5883 & 0.795753 & 1 \\
\hline 13 & 412.1489 & 4025855 & 98.833 & 316.661 & 0.876537 & 3 \\
\hline 14 & 639.5815 & 9003344 & 118.6462 & 3243.304 & 2.251883 & 2 \\
\hline 15 & 376.3808 & 2785671 & 86.03025 & 647.0868 & 1.311196 & 2 \\
\hline \multicolumn{2}{|c|}{$\begin{array}{l}\text { No. of test } \\
\text { images }\end{array}$} & \multicolumn{2}{|c|}{$\begin{array}{l}\text { With unorganized } \\
\text { database }(\mathrm{sec})\end{array}$} & \multicolumn{3}{|c|}{$\begin{array}{l}\text { With organized database as } \\
\text { per indexes }(\mathrm{sec})\end{array}$} \\
\hline 200 & \multicolumn{3}{|c|}{0.0721} & \multicolumn{3}{|l|}{0.0622} \\
\hline 16 & 435.8933 & 4651435 & 103.3007 & 822.7214 & 1.37384 & 3 \\
\hline 17 & 369.8076 & 2818062 & 87.2946 & 258.6729 & 0.836349 & 3 \\
\hline 18 & 266.0732 & 1406845 & 72.71474 & 437.977 & 1.282995 & 2 \\
\hline 19 & 441.7652 & 7186791 & 127.5474 & 537.809 & 1.103363 & 1 \\
\hline 20 & 450.2947 & 4146650 & 95.96222 & 1295.909 & 1.696441 & 3 \\
\hline 21 & 405.7415 & 3655038 & 94.91202 & 237.0266 & 0.764318 & 3 \\
\hline 22 & 414.6593 & 4291438 & 101.7316 & 1138.657 & 1.657108 & 3 \\
\hline 23 & 439.04 & 5612504 & 113.0645 & 1007.022 & 1.514494 & 1 \\
\hline 24 & 569.4626 & 7267216 & 112.967 & 1253.937 & 1.483902 & 1 \\
\hline 25 & 388.5733 & 4062368 & 102.2476 & 810.9251 & 1.444621 & 3 \\
\hline
\end{tabular}

TABLE V. Average CPU Time

TABLE VI. K-MEANS AND RBFNN CLUSTERS

\begin{tabular}{llll}
\hline \hline & Group I & Group II & Group III \\
\hline K-means & 80 & 277 & 247 \\
RBFNN & 82 & 256 & 266 \\
\hline
\end{tabular}


TABLE VII. A: GROUP CHARACTERISTICS

\begin{tabular}{llll}
\hline \hline $\begin{array}{l}\text { Group } \\
\text { Index }\end{array}$ & $\begin{array}{l}\text { Area } \\
\left(\mathrm{mm}^{2}\right)\end{array}$ & $\begin{array}{l}\operatorname{Imax} \\
\left(\mathrm{mm}^{4}\right)\end{array}$ & $\begin{array}{l}\text { RGy } \\
(\mathrm{mm})\end{array}$ \\
\hline & 200.022 & $\begin{array}{l}5157372.515 \\
8525384.26\end{array}$ & $\begin{array}{l}101.726672- \\
177.111404\end{array}$ \\
1 & 624.117 & & \\
& & 16329.42748 & $8.960133-$ \\
& 79.676 & 14331267.47 & 156.117258 \\
2 & 834.711 & & \\
& & & \\
3 & 167.320 & 2818062.292 & $83.6197-$ \\
& 514.583 & 5126772.995 & 151.046937 \\
\hline
\end{tabular}

TABLE VII. B: GROUP CHARACTERISTICS

\begin{tabular}{llll}
\hline \hline $\begin{array}{l}\text { Group } \\
\text { Index }\end{array}$ & $\begin{array}{l}\mathrm{Imin} \\
\left(\mathrm{mm}^{4}\right)\end{array}$ & $\begin{array}{l}\mathrm{RGx} \\
(\mathrm{mm})\end{array}$ & $\begin{array}{l}\text { Approximate } \\
\text { shape }\end{array}$ \\
\hline \multirow{2}{*}{$\begin{array}{l}2.143216- \\
7720.379626\end{array}$} & $\begin{array}{l}0.077902- \\
3.920185\end{array}$ & $\begin{array}{l}\text { Almost Round, } \\
\text { oblong or } \\
\text { rectangular }\end{array}$ \\
& $\begin{array}{l}0.000004- \\
2\end{array}$ & $0.000121-$ & $\begin{array}{l}\text { Oval shaped, } \\
\text { small sized }\end{array}$ \\
& 3992.773763 & 2.436946 & $\begin{array}{l}\text { Triangular } \\
\text { shaped and } \\
\text { other shapes }\end{array}$ \\
\hline \hline
\end{tabular}

TABLE VIII. Performance Evaluation Measures

\begin{tabular}{ll}
\hline \hline Training Accuracy & $95.4 \%$ \\
Classification Error & 0.56033 \\
Entropy & 0.99395 \\
\hline \hline
\end{tabular}

TABLE IX. Classification EFFiciency Evaluation Metrics

\begin{tabular}{llll}
\hline \hline & C1 & C2 & C3 \\
\hline Accuracy & 0.9719 & 0.9686 & 0.9669 \\
Precision & 0.88889 & 0.92857 & 1 \\
Recall & 0.9 & 1 & 0.92806 \\
Specificity & 0.98286 & 0.94693 & 1 \\
F-measure & 0.89441 & 0.96296 & 0.96269 \\
\hline \hline
\end{tabular}

\section{CONCLUSIONS}

This paper presented a novel method to identify persons using shape related features of ear. In a nutshell, the significant contributions of this work are:

- Indexes to ears based on their five shape related biometric features.

- Creating and organising template database of ear biometric features with their index values elicited from RBFNN.

- Quick retrieval of the details of the person when test ear image is presented to the system.

However, the limitation of the work is that, though the system is able attach an index to an ear, there seems to be overlapping of attribute values across the groups. This may lead to a kind of uncertainty due to partaking of an ear in multiple groups. To address this issue, the future enhancement could be to develop a fuzzy indexing scheme.

\section{REFERENCES}

[1] Iannarelli, A., "Ear Identification", Forensic Identification Series, Paramount Publishing Company, Fremont, California, 1989.

[2] Mahbubur Rahman et al, "Person Identification Using Ear Biometrics", International Journal of The Computer, the Internet and Management Vol. 15\#2, pp 1-8, 2007.

[3] Chang, K., Bowyer, K.W., Sarker, S., Victor, B., "Comparison and Combination of Ear and Face Machine Image in appearance -Based Biometrics', IEEE Transaction on pattern Analysis and machine Intelligence, vol. 25, no, 9 ,2003.

[4] Sukhdeep Singh, Dr. Sunil Kumar Singla, "A Review on Biometrics and Ear Recognition Techniques", Singh et al., International Journal of Advanced Research in Computer Science and Software Engineering 3(6), pp. 1624-1630, June - 2013.

[5] Mai V, Khalil I, Meli C, "ECG biometric using multilayer perceptron and radial basis function neural networks", 33rd Annual International Conference of the IEEE EMBS Boston, Massachusetts USA, August 30 September 3, 2011.

[6] A. Sulong, Wahyudi and M.D. Siddiqi, "Intelligent Keystroke PressureBased Typing Biometrics Authentication System Using Radial Basis Function Network", 5th International Colloquium on Signal Processing \& Its Applications (CSPA), 2009.

[7] Chatterjee, A., Fournier, R., Nait-Ali, A., Siarry, P., "A Postural Information-Based Biometric Authentication System Employing STransform, Radial Basis Function Network, and Extended Kalman Filtering", Instrumentation and Measurement, IEEE Transactions on (Volume:59, Issue: 12 ).

[8] Anand Viswanathan, S. Chitra, "Optimized Radial Basis Function Classifier for Multi Modal Biometrics", Research Journal of Applied Sciences, Engineering and Technology 8(4): 521-529, 2014 ISSN: 20407459; e-ISSN: 2040-7467.

[9] Ankit Chadha, Neha Satam, Vibha Wali, "Biometric Signature Processing \& Recognition Using Radial Basis Function Network", CiiT International Journal of Digital Image Processing, ISSN 0974 - 9675 (Print) \& ISSN 0974 - 956X (Online) September 2013.

[10] Ashok.J, Rajan.E.G,'Writer Identification and Recognition Using Radial Basis Function", Int. Jour. of Computer Science and Information Technologies, 1(2), 51-57, 2010.

[11] Kunte.R.S and Samuel.R.D.S, "A simple and efficient optical character recognition system for basic symbols in printed Kannada text", SADHANA ,32(5),521-533, 2007.

[12] M.A.Jayaram, Prashanth.G.K., Sachin.C.Patil, "Inertia based ear biometrics: A novel approach", Journal of Intelligent systems, De_Gruiter publishing, Germany, 2015.

[13] Egor P Popov, "Engineering mechanics of solids, Easter economy edition, $2^{\text {nd }}$ edition 1998.

[14] B Arbab Zavar, Mark S Nixon, "Model based analysis for ear biometric", Computer Vision and Image understanding, vol 115, issue 4, P 487-502, 2011.

[15] Braja M. Das, Paul C. Hassler, "Statics and Mechanics of materials", Prentice Hall, 1988.

[16] Yang Mingqiang, Kpalma Kidiyo1, Ronsin Joseph, A Survey of shape feature extraction techniques, Pattern Recognition Techniques, Technology and Applications, availabl at: www.intechopen.com, pp149.

[17] Riyadh A.K. Mehdi, "The Effect of Dimensionality Reduction on the Performance of Software Cost Estimation Models International Journal of Engineering and Innovative Technology (IJEIT) Volume 4, Issue 9, March 2015.

[18] Shivpuje Nitin Parappa, DR. Manu Pratap Singh, "Conjugate descent of gradient descent radial basis function for generalization of feed forward neural network", International Journal of Advancements in Research \& Technology, Volume 2, Issue 12,ISSN 2278-7763, Dec. 2013. 